\title{
RecRoute: Uma Proposta de Aplicativo para Recomendação de Rotas de Ônibus Utilizando Informações Contextuais dos Usuários
}

\author{
Adriano de Oliveira Tito, Arley Ramalho Rodrigues Ristar, Luana Martins dos \\ Santos, Luiz Antonio V. Filho, Patrícia Restelli Tedesco e Ana Carolina Salgado
}

Centro de Informática - Universidade Federal de Pernambuco (UFPE)

50.733-970 - Recife - PE - Brasil

$\{$ aot, arrr2, lms 7, lavf, pcart, acs\}@cin.ufpe.br

\begin{abstract}
The traffic in major cities has led public transportation to become inefficient, having as one of its characteristics the absence of information to users. In this scenario, Public Transportation User Information Systems emerge as a possible solution, aiming to provide information to passengers and support their decision-making. This work describes our proposal for a system that considers contextual information of both users and traffic to recommend bus routes and support passengers in their decision-making. Thus it is expected to provide information of great relevance for the users of urban public transportation, improving the quality of public transportation by bus.
\end{abstract}

Resumo. O tráfego nas grandes cidades tem contribuído para a ineficiência do transporte público. Um dos principais problemas neste sentido é a ausência de informações aos usuários. Neste cenário surgem como uma possível solução os Sistemas de Informação aos Usuários do Transporte Coletivo que tem por objetivo fornecer informações aos passageiros e apoiar suas decisões. Este trabalho apresenta a proposta de um sistema que considera informações contextuais dos usuários e do trânsito para recomendar rotas de ônibus a esses passageiros, apoiando-os em suas tomadas de decisão. Com isso esperase prover informações de grande relevância para os usuários de transporte coletivo urbano, melhorando a qualidade do transporte público por ônibus.

\section{Introdução}

O trânsito nas grandes cidades, principalmente em países em desenvolvimento, vem piorando diariamente. Com o aumento do número de carros particulares nas vias, os congestionamentos tornaram-se cada vez mais frequentes. Segundo [Zhang et. al 2011] não será possível melhorar o trânsito alargando ruas, construindo viadutos ou fazendo rodízio de carros. Para isto, é preciso que o poder público e as empresas operadoras do transporte coletivo aprimorem o serviço prestado, fazendo com que os usuários utilizem o transporte público [Pilon 2009]. De acordo com [Cutolo 2003], as principais barreiras para a utilização do transporte público por ônibus são a ausência de informações relativas aos serviços e/ou sua baixa qualidade.

Neste contexto, os Sistemas Inteligentes de Transporte - SIT têm por objetivo aplicar tecnologias para apoiar a infraestrutura e melhorar a qualidade dos sistemas de 
transporte [Gómez et. al 2009]. Uma das subáreas dos SIT são os chamados Sistemas de Transporte Público Avançados, voltados ao transporte público. Nesta categoria se inserem aplicações denominadas de Sistemas de Informação aos Usuários, que têm por objetivo prover informações aos passageiros como, por exemplo, o tempo de espera na parada e as rotas de ônibus. Estas informações ajudam os usuários a definir seus trajetos e planejar melhor os deslocamentos [Sussman 2005].

Neste artigo é apresentada a proposta de um aplicativo, denominado RecRoute, para recomendação de rotas de ônibus aos usuários de transporte público, baseado em informações contextuais estáticas e dinâmicas dos usuários e do tráfego urbano. Esta ferramenta procura auxiliar o passageiro em seus deslocamentos, por meio de transporte público coletivo por ônibus, oferecendo informações em tempo real, levando em consideração o deslocamento dos veículos, características dos passageiros e os fatores dinâmicos que podem afetar o transporte como, por exemplo, a situação climática, entre outros. Espera-se que este aplicativo possa auxiliar a infraestrutura do transporte coletivo urbano, melhorando sua qualidade e incentivando pessoas a utilizar o ônibus como opção de transporte público.

\section{Conceitos Básicos}

Nesta seção são apresentados alguns conceitos de Sistemas Inteligentes de Transporte e de Contexto Computacional. Este referencial teórico é necessário ao melhor entendimento desta proposta.

\subsection{Sistemas Inteligentes de Transporte}

Os SIT surgiram nos Estados Unidos no final dos anos 80, por intermédio de um grupo que almejava proporcionar uma nova visão aos sistemas de transportes do país. Os congestionamentos, a segurança, o meio-ambiente e a produtividade foram os principais temas considerados por este grupo [Sussman 2005]. Assim, o desejo de manter uma mobilidade urbana ascendente aliada a uma sustentabilidade ambiental e econômica fez surgir o conceito de Intelligent Vehicle-Highway Systems, posteriormente conhecidos como SIT.

Estas ferramentas integram informação, métodos de comunicação e tecnologias a fim de subsidiar o sistema de transporte de determinada região, integrando pessoas, estradas e veículos, aumentando a eficiência, segurança e uso das vias das redes de transportes atuais. Outros benefícios, relacionados ao meio ambiente, podem ser obtidos através da utilização de SIT, tais como diminuição de congestionamentos e dos índices de poluição do ar. Com a melhor fluidez do trânsito, as emissões de gases serão diminuídas, reduzindo os impactos ambientais [Pilon 2009].

De acordo com os conceitos apresentados, percebe-se que a aplicação de tecnologias no transporte urbano por meio de sistemas SIT proporciona benefícios diretos e indiretos para os passageiros, para as empresas operadoras, para os organismos gestores e para a sociedade em geral.

\subsection{Contexto Computacional}

Contexto computacional pode ser visto como um conjunto de condições e influências relevantes à aplicação e que tornam uma situação única e compreensível [Brézillon 
1999]. Os Sistemas Sensíveis ao Contexto são aplicações que se adaptam sem intervenção explícita do usuário, ou seja, levam em conta informações da situação onde o usuário está inserido para oferecer melhores serviços, aumentando assim sua usabilidade e efetividade [Baltrunas et. al 2011].

Segundo [Vieira et. al 2009], o contexto da interação entre um agente e uma aplicação, para executar uma tarefa, é o conjunto de elementos contextuais instanciados que são necessários para apoiar a tarefa atual, e um elemento contextual é qualquer dado, informação ou conhecimento que permite caracterizar uma entidade em um domínio.

Com o uso do contexto computacional, diversos aplicativos inteligentes podem ser criados, e serem capazes de se adaptar a determinada situação ou fornecer serviços mais relevantes, conforme o conjunto válido de informações contextuais dinâmicas, estáticas ou inferidas e suas interações [Baldauf 2007].

\section{A Proposta do Sistema RecRoute}

O RecRoute terá o objetivo de facilitar o dia a dia das pessoas que utilizam transporte público, recomendando rotas aos passageiros, em tempo real, baseadas em informações estáticas e dinâmicas de contexto relacionadas aos usuários e aos próprios meios de transporte. A Figura 1 representa a arquitetura proposta e que ainda está em desenvolvimento, mas já com alguns módulos em funcionamento. Após ser totalmente implementado o RecRoute fará parte do Middleware do projeto Ubibus [Vieira et al. 2011]. O Ubibus é um Sistema de Transporte Inteligente que oferece acesso a informações de transporte público, em tempo real, baseado em informações dinâmicas de contexto relacionadas aos próprios meios de transporte.

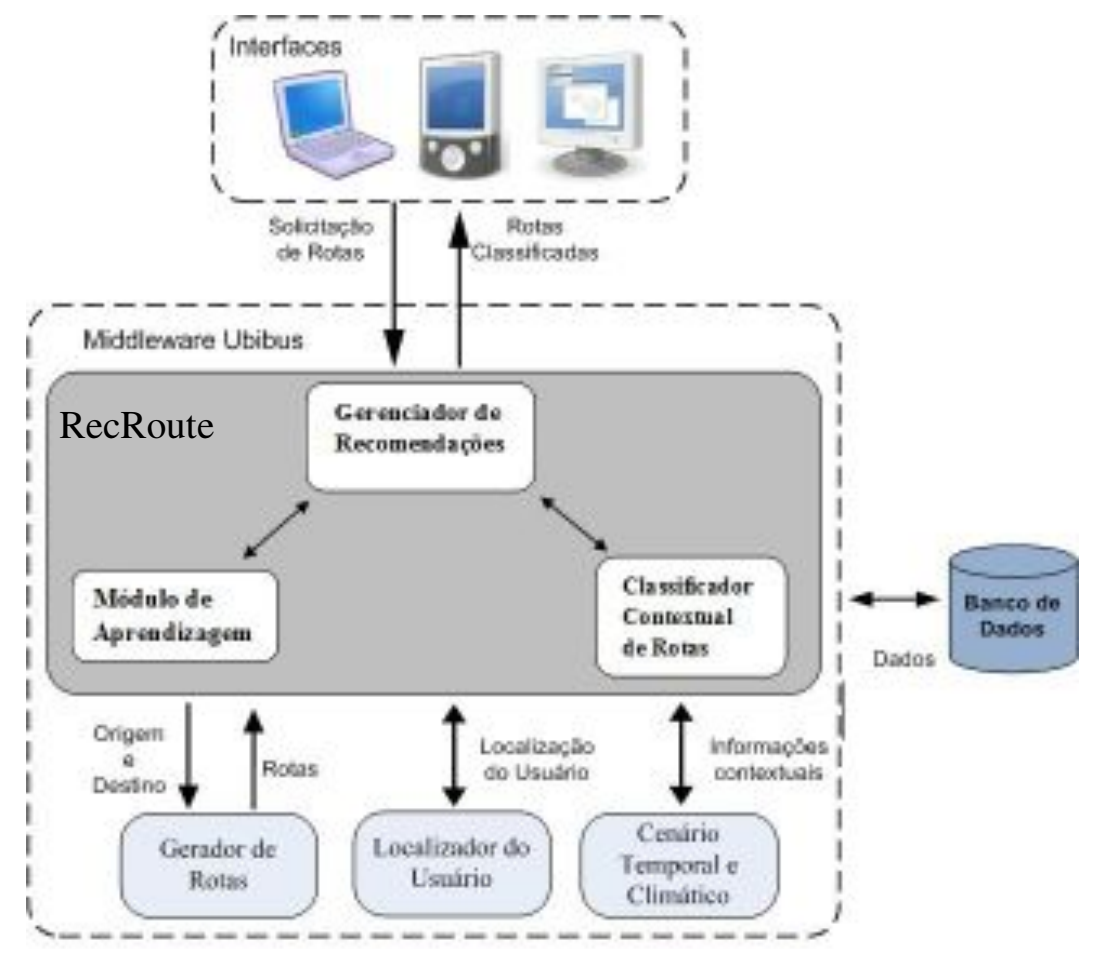

Figura 1. Arquitetura Proposta 
Estão presentes na arquitetura do RecRoute três componentes que desempenham as funções principais ao fornecimento das recomendações são eles: o Gerenciador de Recomendações, o Módulo de Aprendizagem e o Classificador Contextual de Rotas. Outros elementos externos ao aplicativo, mas contidos no Middleware do projeto Ubibus, também serão utilizados nas recomendações das rotas.

A camada superior da arquitetura representa as interfaces que poderão se comunicar com os outros módulos do RecRoute por meio da Web ou dispositivo móvel com sistema operacional Android. A interface Mobile tem o grande benefício de ser portátil, permitindo assim que o usuário possa tomar sua decisão sobre a rota a seguir de qualquer lugar, mesmo que não esteja na parada de ônibus ou em casa. Por esta interface é possível utilizar a posição georeferenciada do dispositivo para gerar mais informação contextualizada sobre o usuário.

A arquitetura possui um Gerenciador de Recomendações, este componente será responsável pela integração dos demais componentes e orquestração do fluxo de execução do processo de recomendação, gerenciando assim, todas as ações do RecRoute como, por exemplo, a entrada e saída de informações, o acionamento de outros componentes da arquitetura do aplicativo e o acesso à serviços do Middleware Ubibus. Será por meio deste elemento que todas as requisições realizadas pelas Interfaces terão acesso às funcionalidades do RecRoute.

O Módulo de Aprendizagem será responsável por gerar e manter um modelo de conhecimento que será utilizado na recomendação das rotas. A formação deste modelo consiste da utilização de técnicas de Aprendizagem de Máquina e Predição [Mitchell 1997] e Algoritmos de Classificação. Inicialmente a aprendizagem foi realizada por meio de um conjunto de registros, denominado conjunto de treinamento, contento informações sobre as preferências e perfis de usuários, informações temporais e climáticas, opções de rotas, e seus rótulos de classes associados, estas informações foram adquiridas junto aos usuários do transporte público, por meio de questionário eletrônico. A aprendizagem se dará de forma supervisionada e posteriormente será mantida de forma incremental, de acordo com o Feedback dos usuários, durante as recomendações.

O Classificador Contextual de Rotas será o componente responsável pela ordenação das rotas utilizando o modelo do conhecimento produzido pela aprendizagem. As rotas serão classificadas por suas características considerando o ponto de origem e destino escolhidos pelo usuário. Após a ordenação, as rotas seguirão para o Gerenciador de Recomendações para serem exibidas aos passageiros, neste caso as melhores rotas entre as opções serão ordenadamente apresentadas nas interfaces.

Conforme descrito anteriormente outros serviços presentes no Middleware do projeto Ubibus serão utilizados pelo sistema de recomendação. Abaixo são descritos cada um deles:

Gerador de Rotas: Este componente, presente no Middleware do Ubibus, tem o objetivo de gerar um conjunto de rotas de acordo com pontos de origem e destino passados pelo usuário. Elementos contextuais do estado atual do trânsito tais como, engarrafamentos, horários de tráfego intenso, acidentes, alagamentos, informações de redes sociais, entre outras informações são utilizadas para gerar as rotas. Localizador 
do Usuário: Tem o objetivo de obter informações contextuais dinâmicas sobre a localização geográfica do usuário que está realizando a requisição de rota. Estas informações serão utilizadas pelo RecRoute durante o processo de recomendação.

Cenário Temporal e Climático: Informações contextuais sobre o tempo e o clima são importantes para o sistema de recomendação de rotas, pois algumas preferências dos usuários podem variar de acordo com estas informações. Este componente é responsável por fornecer informações climáticas e do tempo, que serão utilizadas pelo RecRoute durante as recomendações.

Após a escolha da melhor rota por parte do usuário, o Módulo de Aprendizagem será acionado pelo Gerenciador de Recomendações, a fim de atualizar implicitamente o modelo do conhecimento para os pontos de origem e destino em questão. Tal funcionalidade utilizará técnicas de Realimentação de Relevância (do inglês, Relevance Feedback), bastante utilizadas em sistemas de recomendação e servem para atualizar automaticamente os interesses especificados no perfil do usuário ao longo do tempo, a partir das avaliações de itens recomendados ou por meio da interação entre o usuário e sistema [Cotter e Smyth, 2000]. Esta medida terá por objetivo tornar o sistema mais adaptativo e manter a qualidade das recomendações.

\section{Trabalhos Relacionados}

Foram encontrados na literatura alguns sistemas de recomendação de rotas de ônibus, como: PECITAS [Tumas e Ricci 2009] que considera as preferências dos usuários em suas indicações e UbibusRoute [Lima et al. 2012] que utiliza informações do trânsito oriundas de redes sociais como fonte de informações contextuais.

Analisando os trabalhos citados observou-se que apenas um tipo de informação é considerada durante as indicações (preferências do usuário ou situação do trânsito), não utilizando outras informações dinâmicas importantes, como por exemplo, clima e feedbacks dos usuários.

O RecRoute contempla a captura e processamento de dados dinâmicos dos usuários, do trânsito e ambientais, a fim de prover informações e rotas de ônibus mais realistas e adaptadas às reais necessidades dos usuários do transporte público urbano por ônibus.

\section{Conclusão}

Atualmente os SIT estão sendo utilizados como forma de superar alguns desafios na área de transportes das grandes cidades. A integração entre diversas tecnologias modernas vem permitindo um avanço significativo nesses sistemas. Sendo assim, há uma crescente procura por sistemas cada vez mais dinâmicos e sensíveis ao contexto. A implementação de um SIT sensível ao contexto no provimento de informações aos usuários de transporte público urbano pode proporcionar maior atração dos passageiros e um melhor apoio à infraestrutura do serviço.

Este trabalho teve por objetivo apresentar a proposta de um sistema para recomendar rotas aos usuários de transporte público, capaz de obter e processar informações contextuais dinâmicas e estáticas dos usuários e do trânsito. 
O RecRoute está em fase final de desenvolvimento e, após concluído, será integrado ao projeto Ubibus, um Sistema Inteligente de Transporte que tem por objetivo fornecer aos usuários informações contextualizadas por meio de colaboração coletiva do conhecimento.

\section{Referências Bibliográficas}

Baldauf, M., Dustdar, S., Rosenberg, F. (2007) "A survey on context-aware systems". International Journal of Ad Hoc and Ubiquitous Computing, 2(4): pp.263-277.

Baltrunas, L. (2011) "Context-Aware Collaborative Filtering Recommender Systems". 172 f. Tese (Phd em Ciências da Computação), Universidade de Bolzano. Bolzano, Itália.

Brézillon, P. (1999) "Context in Artificial Intelligence: IA Survey of the Literature". Computer \& Artificial Intelligence 18, pp. 321-340.

Cotter, P., Smyth, B. (2000) "PTV: Intelligent Personalized TV Guides". In: 12th Innovative Applications of Artificial Intelligence Conference. Austin, Texas, USA, pp. 957-964.

Cutolo, F. A. (2003) "Diretrizes para sistema de informação ao usuário". In: 3th Seminário Internacional PROMOTEO, Porto Alegre, RS, Brasil, 2003.

Gómez, A., Diaz, G. Bousetta, K. (2009) "ITS Forecast: GIS Integration with Active Sensory System”. In: Information Infrastructure Symposium, GIJS'09, pp. 1-6.

Lima, V., Magalhães, F., Tito, A. O., Santos, R., Ristar, A., Santos, L., Vieira, V., Salgado, A. C. (2012) "UbibusRoute : Um Sistema de Identificação e Sugestão de Rotas de Ônibus Baseado em Informações de Redes Sociais". In: 8th Simpósio Brasileiro de Sistemas de Informação, São Paulo, Brasil. pp. 516-527.

Mitchell, T. (1997) “Machine Learning”. New York: McGraw-Hill.

Pilon, J. A. (2009) "Sistema de Informação ao Usuário do Transporte Coletivo por Ônibus na Cidade de Vitória-ES". 125 f. Dissertação (Pós-Graduação em Engenharia de Produção) - Universidade Tecnológica Federal do Paraná - Ponta Grossa-PR.

Sussman, J. (2005), "Perspectives on Intelligent Transportation Systems". New York, USA: Springer.

Tumas, G., Ricci, F. (2009) "Personalized mobile city transport advisory system". In: Information and Communication Technologies in Tourism 2009, Amsterdam, Netherlands, pp. 173-183

Vieira, V., Tedesco, P., Salgado, A. C. (2009) "Modelos e Processos para o Desenvolvimento de Sistemas Sensíveis ao Contexto". Jornadas de Atualização em Informática (JAI'09). Porto Alegre, RS, Brasil, pp. 381-431.

Vieira, V., Caldas, L., Salgado, A. C. (2011) "Towards an ubiquitous and context sensitive public transportation system". In 4th International Conference on Ubimedia Computing, São Paulo, SP, Brasil.

Zhang, J., Wang, F., Wang, K., Lin, W., Xu, X., Chen, C. (2011) "Data-Driven Intelligent Transportation Systems: A Survey". IEEE Transactions on Intelligent Transportation Systems, pp. 1624-1639. 